Beziehungen hätte zur Zeitschrift Betrifft Justiz, ob er den habe bitten können, noch einmal nachzuhaken bei dem Stuttgarter Verlag. Der Richter hat gehört, die in Stuttgart hätten sich noch nicht einigen können über diesen Artikel, er werde aber gern morgen seinen Stuttgarter Bekannten noch einmal anrufen und dem noch einmal klarmachen, wie wichtig es wäre, daß dieser Aufsatz in Betrifft Justiz erschiene. Der Beamte Fink bedankte sich und notierte, daß er seinem Anwalt morgen mitzuteilen habe, der unter des Anwalts Namen dort eingereichte Artikel werde in Stuttgart lebhaft diskutiert, die Aussichten seien nicht schlecht. Richter Uschkurat werde dafür sorgen, daß sie noch besser würden. Da der Artikel schon von der Redaktion Kritische Justiz abgelehnt und dem Anwalt mit Floskeln zurückgeschickt worden war, hatte der Beamte Fink Angst, der Anwalt könne durch solche Mißerfolgserlebnisse die Lust verlieren. Er mußte seinen Anwalt Erfolg erleben lassen, also einen Artikel, den er mit dem Anwalt entwickelt hatte, in einer angesehenen Zeitschrift unterbringen. Er mußte für Vorgänge sorgen, für Bewegung, daß der Anwalt erlebte: Es tut sich etwas, der Fall lebt. Zum Glück konnte er seinem Anwalt einen Tag später melden, was Georg Gelter, der Kämpferische, gemeldet hatte: Imprimatur bringt's. Und Imprimatur brachte es. Fink holte das Paket mit zwanzig Exemplaren am Hauptbahnhof ab. Die Winteroffensive lief an. Gierig graste er den Text ab. Gelter hatte doch noch geändert. Leibniz hatte dringend geraten, in dem Text das Wort Opfer durch der Geschädigte zu ersetzen. Gelter hat Opfer vorgezogen. Franz Karl Moors Kommentar: In der FAZ wär' der Artikel Gold, aber in Imprimatur ... Und blies ein unsichtbares Stäubchen von seiner Handfläche...«

Aus: Martin Walser, Finks Krieg, Roman, Suhrkamp Verlag 1996, S. 95 f.

\title{
Monika Frommel \\ Das Kreuz mit der Trennung von Staat und Kirche
}

Im Kirchlichen Amtsblatt für die Diözese Mainz wurden im Dezember r 995, kurz vor Weihnachten, die vorläufigen Bischöflichen Richtlinien für katholische Schwangerschaftskonfliktberatungsstellen abgedruckt. Sie enthalten in $\$ 6$ den interessanten Hinweis, daß es wweder mit dem Selbstverständnis katholischer Beratungsarbeit noch mit dem Schutzkonzept der Beratungsregelung vereinbar « sei, „Ratsuchende auf Ärzte, Krankenhäuser oder Einrichtungen hinzuweisen, die Schwangerschaftsabbrüche vornehmen«.

Nun hätte niemand erwartet, daß ausgerechnet katholische Einrichtungen solche Dienstleistungen erbringen, wohl aber, daß sie sich an das reformierte Gesetz halten. Aber auch dies scheint in Zeiten zaghafter Liberalisierungen nicht mehr gewünscht zu sein. Ratsuchende werden zielorientiert beraten. Wenn sie von ihrem Recht auf Ergebnisoffenheit Gebrauch machen, werden katholische Beraterinnen angewiesen, ihr Mißfallen indirekt zum Ausdruck zu bringen. Sie verweigern dann jede Unterstützung beim Ausfüllen der komplizierten Formblätter (Finanzierung des Abbruchs durch die Länder über die Krankenkassen). In der Richtlinie klingt dies so: Mit dem Selbstverständnis der Beratungsarbeit sei es nicht vereinbar, "Anträge zur Finanzierung von Schwangerschaftsabbrüchen auszulegen, auszufüllen oder dabei unterstützend mitzuwirken«. $\$ 4$ geht noch weiter: »Katholische Beratungsstellen lehnen eine Beratung dann ab, wenn für die beratende Person offensichtlich ist, daß 
eine Beratung im Sinne dieser Richtlinien wegen eines bestehenden Zeitdrucks nicht möglich ist.«

Mit anderen Worten: Wenn eine Abtreibung erwogen wird, deuten katholische Beraterinnen dies als erhöhten Zeitdruck, der »eine verantwortliche, echte Beratung unmöglich macht « (so die unten abgedruckten Kommentare der Bischöflichen Pressestelle in Mainz). Leider sieht das neue Gesetz die Möglichkeit vor, ein zweites Beratungsgespräch anzuregen. Dies wird in den Katholischen Richtlinien strategisch ausgebaut und über die Verweigerung des Nachweises der erfolgten Zwangsberatung zur Falle: $\mathbb{S}$ Abs. 3 sieht vor, daß ein Nachweis nicht ausgestellt wird, "wenn die ratsuchende Frau sich nicht auf eine Beratung im Sinn dieser Richtlinien eingelassen hat «, »die ratsuchende Frau der Beratungsstelle gegenüber anonym geblieben ist « oder »die beratende Person die Beratung als noch nicht abgeschlossen ansieht Schwangere werden also in katholischen Beratungsstellen so lange mit Terminen belästigt, bis sie sich den Nachweis bei einer nicht-konfessionellen Beratungsstelle holen. Statt auf ideologische Belehrung wird auf Zeitdruck gesetzt. Eine postmoderne Art, einen Gesetzesauftrag anzunehmen, um staatlich finanziert zu werden, dann aber das Gesetz im eigenen ideologischen Interesse zu verdrehen.

Pro Familia Mainz forderte das Land Rheinland-Pfalz auf, die katholischen Beratungsstellen von der Zwangsberatung auszunehmen und wie Einrichtungen der autonomen Frauenbewegung zu behandeln, sie nämlich nicht mehr finanziell zu unterstützen. Entsprechende Lernprozesse hätten schließlich auch Beraterinnen bei Pro Familia machen müssen. Sie hätten sich in den letzten zwanzig Jahren belehren lassen, daß die $\mathrm{Zwangsberatung} \mathrm{eine} \mathrm{Staatsaufgabe} \mathrm{ist,} \mathrm{die} \mathrm{staatlich} \mathrm{finanziert,} \mathrm{aber}$ auch staatlich reglementiert wird. Kirchlich wie frauenpolitisch ist die Zwangsberatung kein Feld für autonome Politik. Auch die Kirche muß dies akzeptieren. Tut sie es nicht, muß die Trennung von staatlichen und kirchlichen Funktionen nach hundert Jahren zum erstenmal zwangsweise durchgeführt werden.

Die Bischöfliche Pressestelle in Mainz reagiert prompt auf die Vorwürfe und stellte grundsätzlich fest:

„- Selbstverständlich wird auch in katholischen Schwangerschaftskonfliktberatungsstellen die betroffene Frau auf ibren Wunsch anonym beraten. Ebenso selbstverständlich ist aber auch, daß der Beratungsschein eindeutig einer Person zuzuordnen sein muß, das beißt, einen Namen tragen muß. Die Anonymität wird in der Praxis dadurch gewabrt, daß eine andere Mitarbeiterin der Beratungsstelle als die Beraterin den Namen erfragt und einträgt.

- Nicht die beratungswilligen Frauen müssen sich auf die bischöflichen Beratungsrichtlinien einlassen - sie können vielmebr völlig unvoreingenommen die Beratung in Anspruch nebmen -, sondern die katholischen Beratungsstellen haben diese Richtlinien zur Sicherung der Beratungsqualität und im Interesse eines wirksamen Schutzes des ungeborenen Lebens zur Grundlage ibrer Arbeit gemacht. Von einem Verstoß gegen das Persönlichkeitsrecht kann somit unter keinem Gesichtspunkt die Rede sein.

- Nicht bei bestebendem Zeitdruck - ein solcher besteht in der Schwangerschaftskonfliktberatung immer -, sondern wenn der Zeitdruck eine verantwortliche, echte Beratung unmöglich macht, kann eine Beratung aus nabeliegenden Gründen nicht stattfinden.

- Selbstverständlich beachten auch katholische Beratungsstellen das gesetzlich geforderte Prinzip der Ergebnisoffenheit. Ebenso selbstverständlich ist für sie aber auch das verfassung srechtlich zwingende und gesetzlich verankerte Prinzip der Zielorientierung, das beißt, jede Beratung hat den Schutz des ungeborenen Lebens zum Gegenstand. 
- Die katholische Schwangerschaftskonfliktberatung erfüllt in vollem Umfang den gesetzlichen Beratungsauftrag. Sie tut dies so, wie es der Gesetzgeber wünscht, nämlich am Prinzip des Lebensschutzes orientzert und als weltanschaulich geprägtes Angebot innerhalb des Ganzen des Beratungswesens in Deutschland. Deshalb ist sie auch in allen Bundesländern staatlich anerkannt."

Warten wir ab, wie die Länder - Bayern ausgenommen - auf die neue Zumutung reagieren? Vielleicht einigen sie sich darauf, daß bei der $Z$ wangsberatung ein Kruzifix aufgehängt werden darf. Dann wären zwei umstrittene Urteile des Bundesverfassungsgerichts in optimale Konkordanz gebracht. Das scheinbar fortschrittliche Urteil wird reaktionär gewendet und das klerikale Kompromißurteil wird kirchenpolitisch zum Fallbeil. Ganz anders gehen die Uhren in Bayern. Im Mai 1996 wird der Landtag ein Bayerisches Schwangerenhilfeergänzungsgesetz beraten, das im Beratungsteil kirchliche Richtlinien kodifizieren will und im arztrechtlichen Teil offen das reformierte Bundesrecht unterläuft. Es bahnt sich ein interessanter Konflikt an zwischen Bund und Freistaat, wieviel Macht der Kirche direkt und indirekt zustehen darf.

\section{Theo Rasehorn Leserbrief}

Auch Frechheiten, selbst nicht begangene, aber zugetraute, bestraft das Leben. Um so härter, je länger sie zurückliegen. Da hat Günter Frankenberg bei seinem lesensund zustimmenswerten Beitrag, Hüter der Verfassung einer Zivilgesellschaft, meinen fast 20 Jahre zurückliegenden Aufsatz, Aus einer kleinen Residenz. Zum Selbstverständnis des Bundesverfassungsgerichts, ${ }^{1}$ ausgegraben. ${ }^{2}$ Hier hatte ich eine konservative, kleinbürgerliche Mentalität von Verfassungsrichtern sehr polemisch kritisiert. Frankenberg jetzt: "Boshaft die Charakterisierung der Residenz des Rechts Karlsruhe shalb so groß wie der Zentralfriedhof von Chicago, aber doppelt so tote (S. I60). « Das habe ich zwar nicht gesagt - darüber unten -, aber zuzutrauen war es mir natürlich.

Meine Strafe: Heute wird das BVerfG ja wieder heftig und polemisch kritisiert - aber von einer anderen Seite. Gleichwohl kann ich als Kritiker bei einem nicht sehr gründlichen Leser in die Nachbarschaft von Josef Isensee, dem wohl profiliertesten, schärfsten Gegner der neuen fortschrittlichen BVerfG-Rechtsprechung kommen. Bei seiner neuesten »Abkanzelung " hat er im Hinblick auf die Kruzifix-Entscheidung sogar von dem »Eindruck kulturrevolutionärer Provokation « gesprochen. ${ }^{3}$

Dennoch - oder gerade deswegen? - ist Isensee beim 61. Deutschen Juristentag der Vortrag: Bundesverfassungsgericht - quo vadis? übertragen und dieser als »Festvortrag" noch besonders hervorgehoben worden. Auf diesen Sachverhalt habe ich am 10. 4. 1996 - also bevor mir der Frankenberg-Beitrag zugänglich wurde - den DJT mit dem Vermerk hingewiesen, ob hier nicht auch der "Eindruck kulturrevolutionärer Provokation « entstehe. Diesen Weg möchte ich nicht mitgehen. Nach mehr als 30jähriger Mitgliedschaft habe ich meinen Austritt aus dem DJT erklärt.

I In: Daubler/Kusel (Hg.), Verfassungsgericht und Polıtk (1979) S. $149 \mathrm{ff}$.

2 K] 1996, S. 1 (Fn. I)

3 Bildersturm durch Grundrechtsinterpretation, ZRP 1996, 10, 15. 